\title{
SIMULATOR WITH EMBEDDED INTELLIGENCE FOCUSED ON THE DESIGN PROCESS
}

\author{
Arango, I. ${ }^{*} \&$ Herrera, A.** \\ ${ }^{*}$ Department of Mechanical Engineering, EAFIT University, Medellín, Colombia \\ ${ }^{* * *}$ Mechatronic and Machine Design Group, EAFIT University, Medellín, Colombia \\ E-Mail: iarango@eafit.edu.co, aherre14@eafit.edu.co
}

\begin{abstract}
Mechatronic design practice was conceived as various successive steps involving expertise. However, employers expect recently graduated engineers to start working with the shortest training period. This paper reports a research that developed a simulation tool that introduces modifications and additions to the regular methods of dynamic simulation, integrating in it several of the steps of the systematic mechanic design. The design tool encompasses for each element or object of the simulator seven new features that in an intelligent way gives the student a little design practical expertise. The connection between elements follows the method by wires and the window of assembly includes a workspace where the $3 \mathrm{D}$ depiction of all elements is seen and animated according to the values of the variables. The concept was prototyped and now all technological components are available to start the development of a product. This concept, due to the volume of information that it uses, instead of being attractive to cover all fields of knowledge is valuable to adapt to specific fields as academic courses. Potential users evaluated the attractiveness of the concept through a work section, giving good indicators.

(Received in August 2020, accepted in October 2020. This paper was with the authors 2 weeks for 1 revision.)
\end{abstract}

Key Words: Mechatronic Simulation, Engineering Training, Dynamic Systems, Conceptual Design, 3D Animation, Methodical Design

\section{INTRODUCTION}

Systematic engineering design of the technical systems has been dealt with by quite a few researchers [1]. This work responds to the rapidly changing customer demands, ever-intensified marked competition, growing product complexity and demanding legal requirements. There is a high demand on improved production efficiency, product quality, energy consumption and cost containment for manufacturing companies [2]. In order to respond, software has been the most useful tool integrated in the process. The most significant tools are related to calculations, simulation, circuits, FEM (Finite Element Method), CAD (computer-aided drafting), CAM (computer-aided manufacturing), embedded circuit programming and lastly PLM (Product Lifecycle Management). Mechatronics systems such as industrial robots, hybrid vehicles, modern computer numerical control machines, medical instruments and communication and satellite systems have been developed through functional and spatial integration of subsystems with various engineering disciplines [3]. The electro-mechanical design for elements that need to be created from scratch has available many CAD tools with a large number of functionalities to construct models, but the design intended for synthesis, using commercial elements, is just starting. For new concepts, the design procedure users are requiring new tools in which to work simultaneously in all the steps and with emphasis in the synthesis tasks that do not have mature and enough tools [4]. The generation of a range of concepts is often a significant challenge for novice designers given their limited domain knowledge and lack of relevant design experience then the task is to enhance both their understanding of design principles and design capabilities [5].

Design synthesis is a method for the creation of a number of high value alternatives for a specific requirement. The computational tools for synthesis design allow designers to improve 
decision making, by the automatic generation, analysis and evaluation of the possible designs [6]. Simulation modellers study complex problems, built conceptual models, and transform those conceptual models into computer models to represent and study the real world complex phenomenon in a simple manner [7]. To meet industrial requirements, engineering education is constantly revising the programs' courses to include the training of modern advanced virtual prototyping technologies [8].

Simulation started in the Fifties. Some scientists learn to develop software and do their research with the help of their own tools, which later becomes a freely usable or commercial tool due to their usefulness [9]. Below is found a referential summary of the birth of graphic simulation programs, which finally and gradually replace textual code programs in early education which is the focus of this research. The methodology developed in the Sixties by the professor of Engineering Jay W. Forrester [10] is one reference for the analysis of the dynamics of complex systems. The method is used to analyse models in several areas through the graphic representations of the functional elements with interconnections among them to form systems [11, 12]. In 1978, Hilding Elmqvist developed a new approach for the modelling of physical systems through the design and implementation of a graphic language called Dynamic Modelling Laboratory (Dymola ${ }^{\circledR}$ ) [13]. This language uses multiple-object elements. These programming elements allow for direct communication among them, and they offer a physical perspective that takes the user closer to reality [14]. In 1986, the software Laboratory Virtual Instrument Engineering Workbench $\left(\mathrm{LabVIEW}^{\circledR}\right)$ is introduced with the initial objective of obtaining, managing and controlling data by means of a graphic programming language $[15,16]$. It uses functional boxes passed to the design area via drag and drop from toolboxes and then connected via cable. LabVIEW ${ }^{\circledR}$ has mathematical, logical and control components, which allow developing complex systems as well as immediate result display [17]. Likewise, Jack Little, Cleve Moler and Steve Bangert introduced Matrix Laboratory (MATLAB ${ }^{\circledR}$ ) in C language in 1983 as a programming tool with new functions organized in libraries. Subsequently, in 1990, MathWorks ${ }^{\circledR}$ owner of MATLAB ${ }^{\circledR}$ introduced Simulink as an applicative extension of this programming tool. Nineteen years after his first version, in 1997, Hilding Elmqvist presented an update of his work with an object-oriented language called Modelica $^{\circledR}$, which was developed to describe complex physical systems in a simple way, using tools that implement mathematical models and computer simulation [18, 19]. Currently, Modelica ${ }^{\circledR}$ has been used as a platform to develop other simulation tools such as Simulation $\mathrm{X}^{\circledR}$ and MapleSim ${ }^{\circledR}[20]$. Dymola ${ }^{\circledR}$ provides several object-oriented libraries such as mechanical, electrical, electronic, hydraulic, thermal, control systems or process-oriented subcomponents [21]. Currently, "Open Modelica ${ }^{\circledR}$ " is an open-code graphic and modelling simulation environment that is also based on Modelica ${ }^{\circledR}$.

Computer-Aided Design (CAD) and Computer-Aided Engineering (CAE) are disciplines with separate histories and consequently different conventions, data representations and Workflows [5]. Dynamic simulators have been gradually incorporating the capacity to import 3D-files. These ones are created using Computer Aided Design (CAD) and connected in a way that allows for motion, in relation to linear and angular variables. For example, Simscape $\mathbb{R}$, which is an extension of Simulink ${ }^{\circledR}$ working with Simmechanics ${ }^{\circledR}$, a specialized toolbox, part of product family, which uses a physical network approach to model building: components (blocks) corresponding to physical elements that are joined by lines corresponding to the physical connections that transmit energy [22]. Finally, there is an effort to integrate or connect some functions that other tools have and use successfully. For example, CAD (computer-aided design) tools include FEA (finite element analysis) and simulation tools or simulation tools include connection to CAD, or merge two applications as CAD and CAE. Other important advance is the integration of intelligence to the processes of programming the fabrication in CNC machines [23]. 
Despite the advances, there is still work to be done using the capacities available in computer and software, to explore different ways to improve the mechanical and mechatronic design procedures. The concurrent multi-domain system design is complex and challenging. The first aspect of this complexity is related to the fact that companies handle an increasingly considerable amount of data and information from different domains, such as the design, production and maintenance [24]. Students spend hundreds of hours training with simulators that work with little practical knowledge. Employers expect recently graduated engineers to start working with the shortest training period possible, but the volume of information is too broad and the available time to assimilate is too short. Then, engineering students need tools that: can handle the theoretical concepts and at the same time, elements, rules, standards and information of the practical world; while also, integrating other tasks today computationally unconnected but related. In this paper is reported a research that develops a prototype of a simulation tool that includes changes and additions to the regular methods of dynamic simulation with the objective of integrating some tasks belonging to steps of the mechanical and mechatronic design and makes, those interact with a little intelligence. In the second section, the methodology of incorporation is presented; in the third section, the results of a work section are presented; in the fourth section, the conclusions are listed.

\section{METHODOLOGY}

Simulation tools are very advanced, but users without industrial or practical experience often arrive at wrong results using elements that do not connect directly or parameters that do not exist in the market. To bring the results closer to real products, the conceptual and synthesis tools must incorporate more technical information. Therefore, the most productive field of work is to take a simulator and incorporate a little intelligence about conceptual design rules based on the functionality of the elements and plenty of technical information.

The selected methodology, the systematic mechanical design, which is close to that used in mechatronic design for short productions and prototypes that use sub-system integration [25]. An approach to the environment in which an engineer currently performs is achieved through this proposal. An engineer designs a significant percentage of equipment or machines using functional elements obtained in the market. Then every element of a toolbox working with these methodologies and with the proposed concept, presents seven new features: 1) An icon with information concerning the function and the type of energy that it uses. 2) A real commercial reference with its specific parameters. 3) An animated 3D representation. 4) A sensitive identifier of what type of element it is and if elements connected to it are compatible. 5) A protection alarm that indicates when the element's potential variable is experiencing a value greater than the maximum permitted by the commercial parameter. 6) A counter indicating the amount of the energy used and destroyed due to inefficiency. 7) A mathematical representation of the elements based on functional equations. These attributes go in the direction of integrating most of the steps of design in the simulation applications.

\subsection{The icon}

The first information coded in the icon is the type of energy, incoming or crossing the element. For this case, a colour code was added to the group of symbols. This is important in mechatronic design where it is worked with more than one type of energy. Through the system, it crosses matter, energy and information. Then the user can identify the field of knowledge, which the element belongs to. The data and orders are coded black. Light green for linear mechanical, dark green for rotational mechanical, yellow for electrical, red for thermal, light blue for gases and dark blue for liquid are used. Fig. 1 shows the codes including the state of the mass that carry the energy. 


Linear Mechanical E. Thermal E. in a solid Thermal E. in a liquid
Rotational Mechanical E Thermal E. in a gas
Electric E.
Chemical E.
Thermal E.

Figure 1: Colours associated to the types of energy and the state of mass.

\subsection{The function}

The objects are classified according to their function. The colour on the left upper strip indicates the type of energy the element is fed with. Right upper strip colour represents the type of energy the element delivers. Centre of icon has the symbolic representation of the element. A character with one or two letters indicating the type of function of the element is found in the upper strip. The main types of functions used to work in a system are shown in Fig. 2. In this, four elements of each function are listed. Natural and artificial sources of energy (So); Energy converters (C); Mechanical motion converters (Mc); Energy transformers (Tr); Energy accumulators (Ac); Energy switches (Ir); Energy transmitters (Tm); Energy destructors (De). Here, the concept of "destroyed energy" is the energy with potential and flow variables, which cannot permit to be convert this energy into some useful work. Due to the importance of the correct use of energy, energy destruction elements (brakes) and anti-destruction elements have been introduced (bearings, linear guides, ball screws, among others). Finally, in order to test the designs, functions representing forces and torques that are doing some known work.

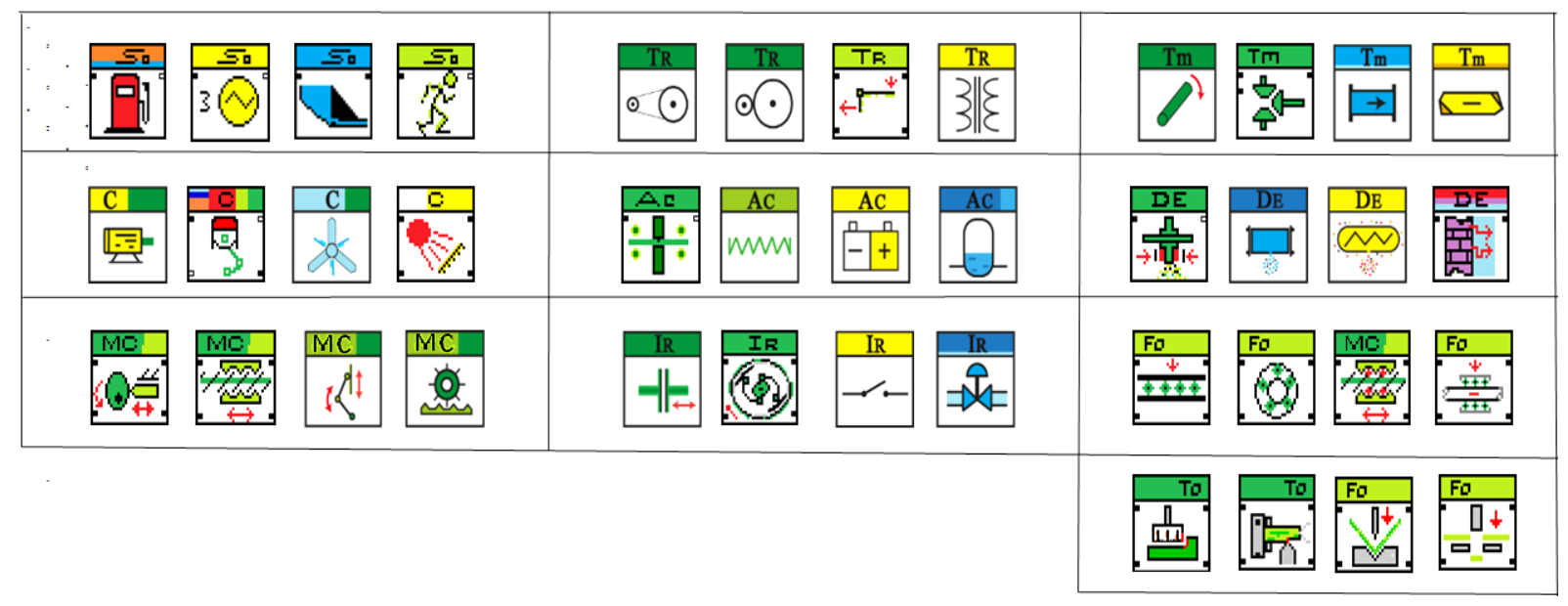

Figure 2: Types of functions and four elements of each.

\subsection{A commercial reference}

In all fields of engineering, the market offers various elements to fulfil each function. In each function, the toolbox presents the options and inside one option, a list of sizes is presented with their corresponding technical characteristics. In addition, recommendations about the type of technology recommended according the application. Many element manufacturers give users applications that help in the selection. Here they are integrated working with other attributes. For example, let us see the selection of a transformer. First, general information about the speed and torque required in the output and the input speed of the application is entered. Other characteristics as type of service, dynamics, ambient, lubrication are included. With it, the object gives recommendations of the type of transformer; for example, a gearbox in a list in which is found belt and pulley, chain and pinion and contact rollers. Clicking in the selector of 
gearbox, other selector with the option worm screw, helical parallel, bevel, pendulum, etc. is presented. Once one of those types of gearboxes is selected, the object delivers characteristics of its technology and efficiency. Then the type of gearbox is selected; for example, a worm screw. The object lists all the references from the database that fulfil parameters. The ratio of transformation estimated from the request is set. The selected reference brings the data of the maximum torque that can safely be connected in the input and the output torque taking into account efficiency.

\subsection{D representation}

A programming object including some pages, list boxes, controls, indicators, graphs, and a 3D representation represents each element. The 3D representation has controls to locate and orientate the element on its own display, and on the 3D display of the common workspace of the simulator where all elements are assembled to configure the general result. See Fig. 3. The point to start for the construction of the moving element is the $3 \mathrm{D}$ file provided by the manufacturer. The element can be as simple as a motor in which the only piece that moves is the shaft; or as complex as a crank-rod mechanism in which there are three pieces moving and their positions are represented by geometric equations that are a function of the angle. Afterwards, the file is converted to the format .wrl or .stl that uses the mathematical editor in order to connect the value of the variables of the element with the motion of the pieces of the elements.
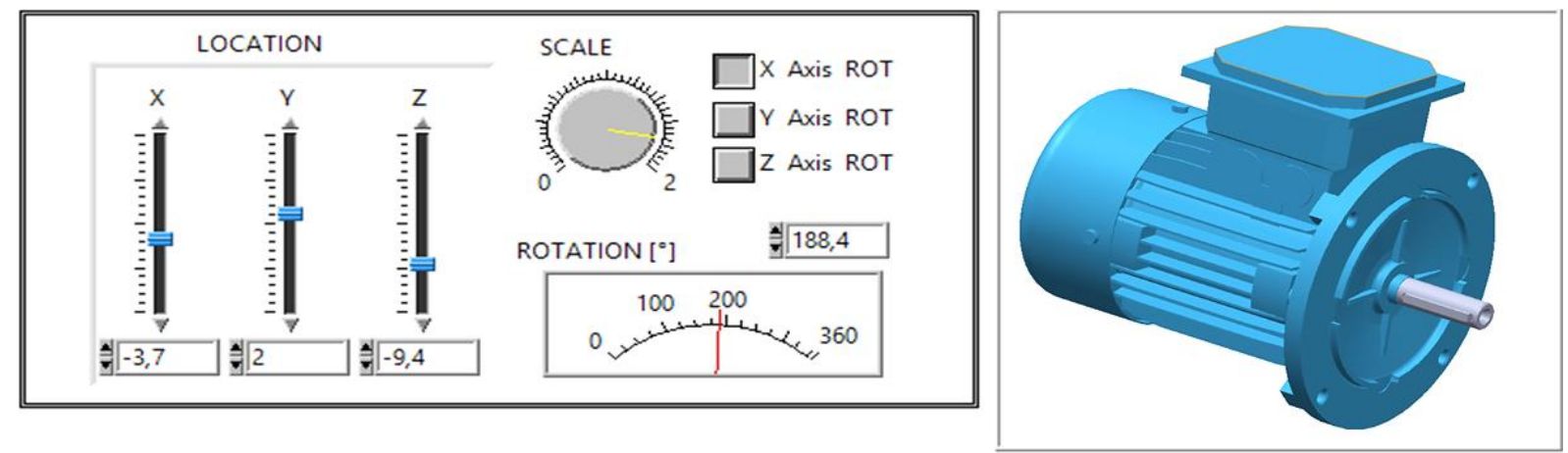

Figure 3: Control to locate and orientate the element on its own and common workspace to be assembled with others.

\subsection{Sensitive identifier}

It is common for programming objects to have numerical, logic and text as primary types of information that determine if one can connect to another. In the engineering world the type of energy is what determines if an element can be connected to other. For example, in rotational mechanics all the elements are connected through shafts. Most simulators allow you to connect elements of different technical systems because the type of mathematical information is compatible. This event generates a design error and the user must be advised for correction improving this way his/her training. In Fig. 4 left, a system constituted of a battery, which is feeding current to a DC motor, is observed. This motor is connected to a torsional spring and from the other side of the spring to a flywheel. Attaching some instruments, this could be the simulation scheme of a machine to measure flywheels moment of inertia. In the same figure, below it is observed the same system but instead of the torsional spring, a compression spring has been connected. This is a functional error because the compression spring belongs to the field of linear energy. In the wires that connect the element it is observed that the simulator does not accept the connection, it presents black, dashed lines and crosses as marker. Those features allow for tests of the conceptual design to be conducted in the simulator. 


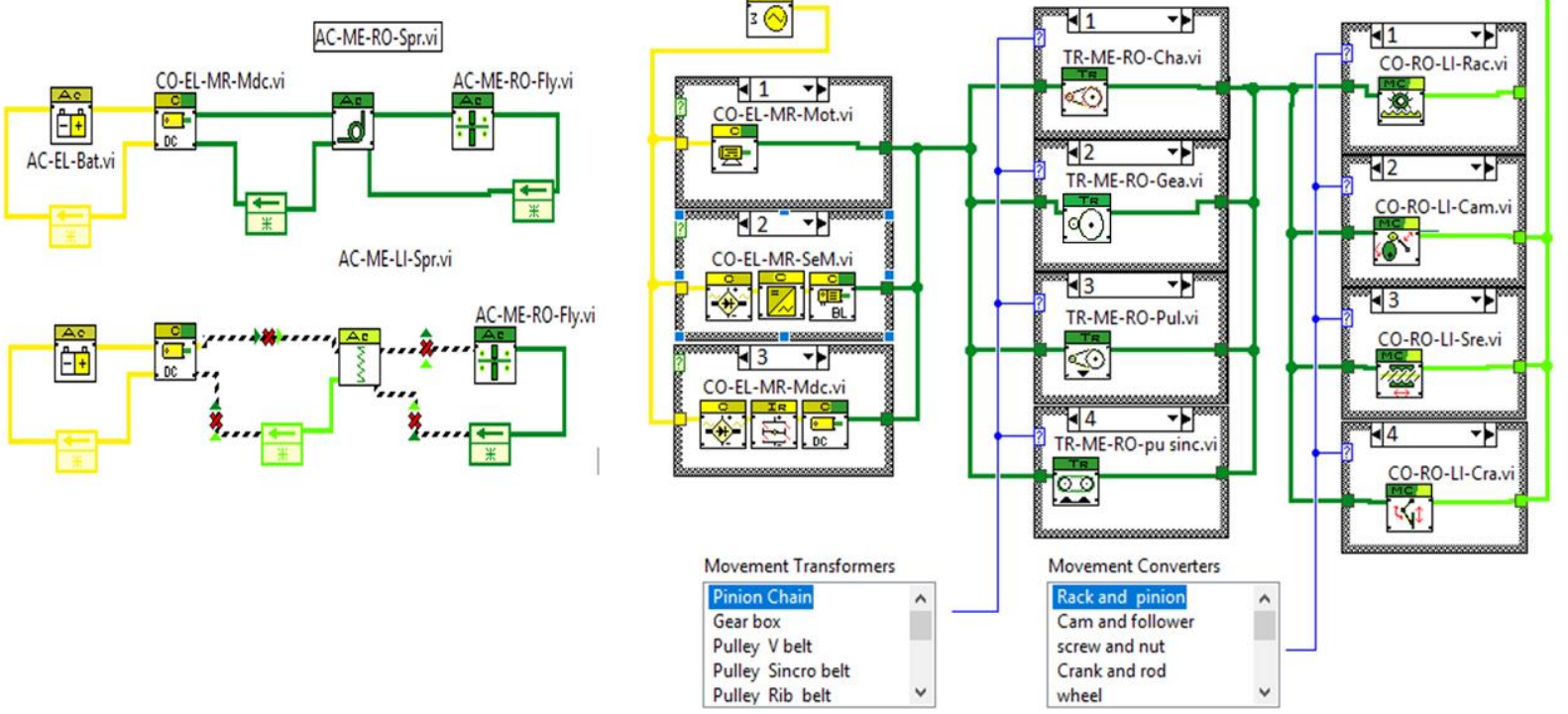

Figure 4: Upper left, good connection; lower left, wrong connection; right, morphologic three.

One of the mandatory tasks in the conceptual stage of the design is the presentation of an array of alternatives (morphologic three) with which the design criteria are evaluated with respect to the client's requirements [26]. This matrix is constructed using the if-else function of the development environment and in this case, the graphic part of each option is in a box so that the evaluation is logical. Suppose the design of a metal mechanical broaching machine has been ordered. Fig. 4 right shows the partial edited scheme in which in the matrix of possibilities are found three motors, four transformers and four converters of linear to rotational motion. With the selector controls, any combination may be chosen. Each element has in its database punctuation in characteristics or criteria as efficiency, relative price, weigh/power ratio, etc. that the application summarizes and displays.

\subsection{Protection}

All the elements of all the technical systems must endure the potential variable. Torque for rotational system, force for a linear, pressure for a fluidic, temperature for a thermal, etc. Size selection of commercial elements is done after finishing the simulation; however, here it is introduced in the simulation. An exceeding value of the potential variable destroys an element. So, the parameter associated with this variable is the one that mainly defines the size of the element. For example, a nail-making machine requires torque to do the work and it is transmitted along the shafts from the motor to the subsystems of feeding, fixing, metal forming and cutting. If the user increases the diameter of the nail wire, then the torque in all tools is increased as well. If in the simulation, the maximum torque of work indicated by the parameter for one of the elements is exceeded, a light turns on warning it. If the user continues increasing the nail diameter little by little the warning of all elements will turn on. This procedure allows finding the exact size of each element to configure a machine for a required nail diameter and validate calculations done by other methods. Now, if in the simulation, we use commercial elements and if the simulator indicates the variable of potential is being exceeded, it allows for selection of the right size or reference of the elements to be selected joining various design steps in one.

\subsection{Indicator of the energy used and destroyed}

Because of the growing interest in the correct use of energy, the operational efficiency of equipment determines its commercial viability. So, in order to analyse efficiency in the 
optimization process, indicators of energy used and wasted in each element have been added. The efficiency of an element varies with the entire range of the potential and flow variables, thus, to calculate the relationship between the energy it receives and the energy it delivers, the tabulated efficiency map of the element is used. The map is saved in a matrix with 4 dimensions (speed, torque, \% load, efficiency). The percentage of load is quasi-parallel lines to the maximum torque. As it can be explained in Fig. 5, the efficiency map is entered by the horizontal axis, the speed axis, then move vertically until the curve of percentage of load, here the efficiency is read. After that, turn left to the Y-axis, the torque axis, where it is read. The used and destroyed energy $\Delta E_{d e s}$ is calculated as a function of the variables of potential $V_{p}$ and flow $V_{f}$; and the efficiency $\eta$, according to the following equation:

$$
\Delta E_{\text {des }}=\left(V_{p-\text { out }} * V_{f-o u t} * \Delta t\right)[1-\eta]
$$

Each map has a closed zone of maximum efficiency, an area where designers try to set the operation point of their machines.

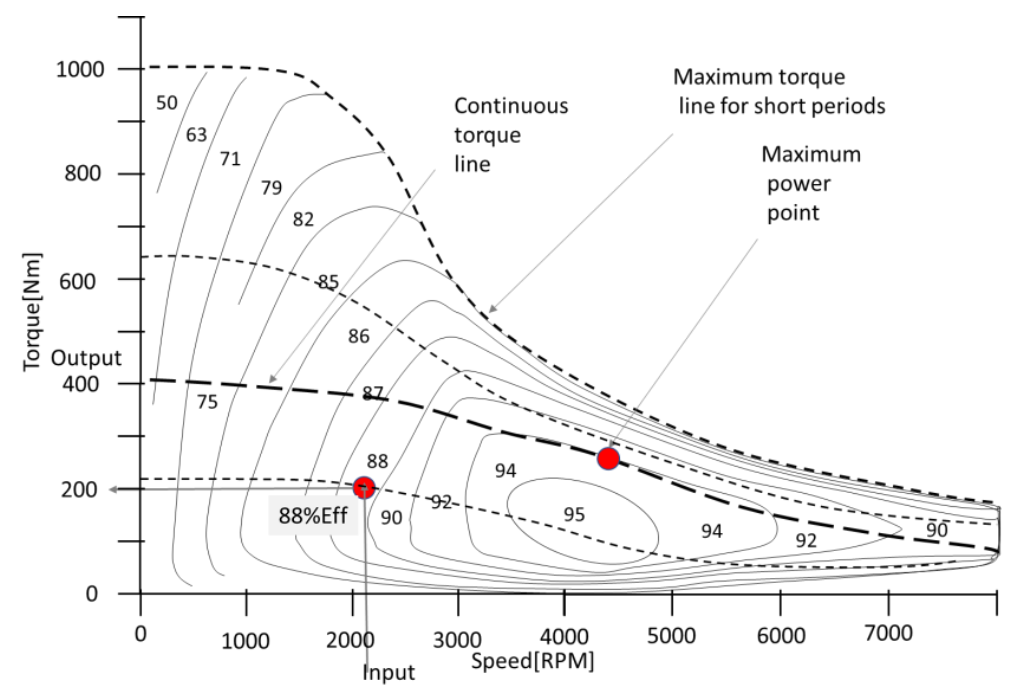

Figure 5: Example of a map of efficiency corresponding to a vehicle's electrical motor.

\subsection{Mathematical representation}

The mathematical solution is not the focus of this research, and then to solve for the changes in the variables of the system through time caused by the interrelation between elements, available solvers in engineering software have been used. In this case, the solver and all the resources of LabVIEW $^{\circledR}$. In the proposition of the simulator, each object has four main dependent variables changing as a function of time. These variables are classified as: potential variable, flow variable [27], summation of inertia moment (or inertia), the integral of the flow variable and the independent variable, the time. To distinguish what changes each element does to the variables, functional equations are used. To obtain the magnitude of the output variables, five equations depending on the variables and the parameters of the elements are multiplied or added to the input variables. The first function takes the moment of inertia of the rotating parts of the element and adds it to the accumulated inertia. The second function calculates the angle that the element has rotated in the delta of the iteration time and adds it to the cumulative angle. The third function is the quantifier of the energy used by the element. The fourth and fifth functions solve the new potential and flow amounts and change according to the type of function of the element, as summarized below.

- Sources of energy. There are not natural sources of electrical or mechanical energy. The artificial sources provide voltage and torque as potential variables.

- Energy accumulators (mechanical spring, kinetic and potential in the mass). 


$$
\begin{gathered}
E_{s p_{-} i}=E_{s p_{-}(i-1)}+\left[0.5 k *\left(\theta_{i}{ }^{2}-\theta_{(i-1)}{ }^{2}\right)\right] \\
E_{k_{-} i}=E_{k_{-}(i-1)}+\left[0.5 I\left(\omega_{i}^{2}-\omega_{(i-1)}{ }^{2}\right)\right] \\
E_{p_{-} i}=E_{p_{-}(i-1)}+m g(\Delta t * v)
\end{gathered}
$$

with $E$ the stored energy, $k$ the spring constant, $\theta$ the angle, $m$ the mass, $I$ the moment of inertia, $\omega$ the rotational speed and $v$ the linear speed.

- Energy converters. Electrical/fluidic/thermal energy in rotational mechanical energy. The efficiency map describes in tables or graphs those equations.

$$
\begin{gathered}
f\left(V_{f_{-} \text {in }}, K t e_{1}, \eta\right)=V_{p_{-} \text {out }} \\
f\left(V_{f_{-} \text {out }}, \text { kte } e_{2}\right)=-c V_{p_{-} \text {in }} \\
V_{f_{-} \text {in }}=f\left(V_{p_{\text {so }}}, R_{1},-c V_{p_{-} \text {in }}\right) \\
V_{f_{-} \text {out }}=f\left(V_{p_{-o u t}}, R_{2},-c V_{p_{-} \text {wo }}\right)
\end{gathered}
$$

with $V_{f_{-} i}$ the flow variable, $V_{p_{-} i_{-}}$the potential variable, $\eta$ the efficiency, $V_{p s o}$ the potential of the source; $-c V_{p-i n}$ the potential caused in the inside by the flow in the outside, $c V_{p-w o}$ potential caused by the work in the outside, $R_{i}$ the resistance to the flow in each side and $K t e_{i}$ parameters of the converter.

- Energy transmitters. Simple and divisor.

$$
\begin{gathered}
T o_{\text {in }} * \eta=T_{\text {out }} \\
T o_{\text {in }} * \eta=T o_{\text {out_1 }}+T o_{\text {out } \_2}
\end{gathered}
$$

- Energy transformers.

$$
\begin{gathered}
T o_{\text {in }} * M A * \eta=T o_{\text {out }} \\
\omega_{\text {in }} *(1 / M A)=\omega_{\text {out }} \\
M A=\emptyset_{\text {out }} / \emptyset_{\text {in }}
\end{gathered}
$$

with $M A$, the mechanical advantage and $\emptyset_{i}$ pulley or gear diameter.

- Motion converters. Rotational to linear.

$$
\begin{gathered}
\left(T o_{\text {in }} / R\right) * M A * \eta=F_{\text {out }} \\
\omega_{\text {in }} * R *(1 / M A)=V_{\text {out }} \\
M A=R_{a} * \Delta \theta / \Delta X
\end{gathered}
$$

with $R_{a}$ the radio, $X$ the linear displacement, $V_{\text {out }}$ the linear speed, $\theta$ the angle. In some of these elements, the $M A$ is a variable as a function of the angle and the geometry of the element, i.e., crank-rod, cams.

- Energy destructors. Pure and regulation with destruction of energy.

$$
\begin{gathered}
T o_{\text {out }}=T o_{\text {in }} * \eta \\
T o_{\text {out }}=T o_{\text {in }}-f\left(V_{f}, R\right)
\end{gathered}
$$

- Energy Switches. Closed and open.

$$
\begin{gathered}
T o_{\text {out }}=T o_{\text {in }} \\
T o_{\text {out }}=0
\end{gathered}
$$

\subsection{Simulator operation}

The magnitudes of the variables of a system are evaluated following the changes each element does to the variables. This procedure takes the functional equation of all the system and evaluates it numerically using a small instant of time. Fig. 6 right shows part of a pumping system that is a graphic example of programming $N$ elements. This system is constituted by three rings, which are differentiated by the type of energy they use: electrical, rotational 
mechanics and fluid (liquid). If we were to solve the system, we write three differential equations and solve it. Here the system is solved by numerical iterations and for that; each ring moves continuously the information internally and from it to the next ring (Fig. 6 centre). The element that connects two rings verifies the neighbouring ring variables to use their magnitudes in the solution of its own equations and vice versa. In the simulation workspace, the system has the feedback wires for each ring (Fig. 6 left).
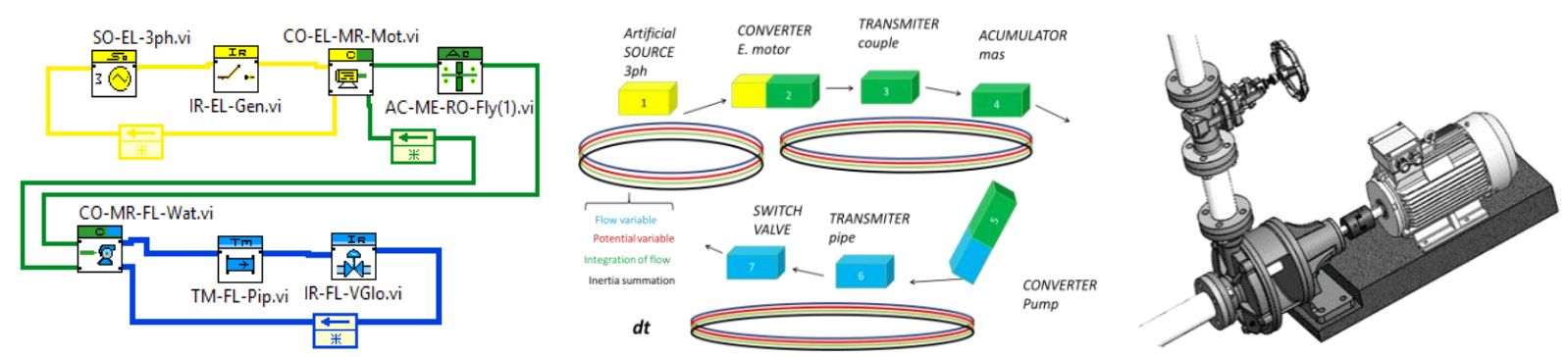

Figure 6: Three aspects of the same system: design scheme, mathematical operation and 3D representation.

To avoid the confusion that might be created by a large number of inexistent lines or wires between elements that do not exist in a real physical system, all lines are correlated packed in only one line of information, in a cluster. To solve the dynamics using numerical methods, Euler's method for didactic demonstrations or high order Runge-Kutta for greater precision tasks are selected. The procedure followed by the user to complete the design of a machine or equipment is the following. The user establishes the functions required. Each function unfolds a list box of elements fulfilling the function. Once, the element has been selected, dragged from the toolbox, drop in the common workspace, wired to the other elements; the front panel of the element can be displayed, and its operational characteristics observed. In one of the pages of the front panel of each element, the controls to locate it and orientate it are found. Once all elements have been placed in the workspace, the simulation is started and the kinematic and dynamic behaviour of each element is observed through its own numerical and graphical indicators.

\section{RESULTS}

Through a workshop, the first perception of users about the tool's usefulness, complexity when setting parameters and ease of use is evaluated. The didactic usefulness of the simulator was tested through a practical work session with students at EAFIT University. The called population was 120 students from the seventh of ten semesters. The sample was 54 students who showed interest in testing the application and had the conditions to handle the tool. With this, the confidence level was $95 \%$ and the margin of error $10 \%$. Candidates selected were those who had knowledge of how graphical programming tools work or with experience using one of the dynamic simulators that work with graphical representations and connections by wires as Modelica ${ }^{\circledR}$, Simulink $^{\circledR}\left(\right.$ MATLAB $\left.^{\circledR}\right)$, LabVIEW $^{\circledR}$, etc.

In the work section, the user was consulted about general functionality, simplicity in parameterization, user-friendliness and the importance of the three-dimensional parts during the design. Two types of questions were formulated: qualitative questions with two groups of answers, one indicating, "Reduces or increases" and another displaying "Yes" or "No". The average of the answers indicates $92.6 \%$ of the students think the application reduces the time to design. In addition, $88.9 \%$ has the opinion that the tool is useful. There are also groups of quantitative questions with answers on a range between 1 and 10. The degree of complexity of the parameterization of the tool got answers with the highest percentage located in numbers under 5, 2 and 4, indicating a good result. The degree of user friendliness of the tool in the task 
of designing got answers with the highest percentage located in numbers 5, 7, 8 and 9 indicating a positive result. The usefulness provided by the 3D model got answers with the highest percentage in the numbers 8,9 and 10 showing that the students perceive a high degree of usefulness.

Nevertheless, some limitations were reported by the students and by the developers of the tool. The most relevant disadvantage of the tool is its limitation to a narrow field of engineering or to a specific activity due to the impossibility of having available the thousands of commercial elements.

\section{CONCLUSION}

The concept and the prototype of a dynamic simulation tool with changes and additions that integrate several of the activities of the mechatronic design process and practical knowledge as one was presented.

In contrast to applications that work in a wide field of knowledge, an application designed for a narrow multi domain field, offers the possibility of embedding secondary training functions for the user.

The functional discrimination of the elements and/or objects together with the colour for each type of energy, works as a tutor based on analogies. The inclusion of colours to differentiate the type of energy; the name of the function in the icon; the acceptance of wiring only between elements of the same class; the alarm due to overload of an element and the 3D illustration of the commercial element to which it corresponds; are characteristics that indicate a significant intelligence. This intelligence gradually, with its use, trains the user.

In terms of programming, the creation of the classes according to the type of energy; the declaration of the objects as members of different classes; the inclusion of technical information; and the development of a mathematical model of the elements as programming objects; had a positive contribution in the performance of the synthesizing tasks in the design procedure.

The student finds it difficult to work with the interfaces between elements, especially when the types of energy change, but if the element that seeks to connect to another indicates that it is not possible, it forces a deductive or inductive process that results in a correct selection, resulting in learning.

The 3D illustration of the commercial element gives the user an approach to the industrial world. The student knows the qualitative or schematic differences, but not visually and here, he/she connects the illustration with the information.

The overload alarms in the potential variable allow the design process to focus first on the concept, then on the kinematics and finally on the dynamics and with the help of those alarms, the elements are dimensioned in a short time.

The concept was prototyped and now all technological components are available to start the development of the product.

\section{ACKNOWLEDGEMENT}

This work was partially supported by the Colombian Organization for Scientific Research, COLCIENCIAS, grant number 033-2018.

\section{REFERENCES}

[1] Börklü, H. R.; Yüksel, N.; Cavdar, K.; Sezer, H. K. (2018). A practical application for machine design education, Journal of Advanced Mechanical Design, Systems, and Manufacturing, Vol. 12, No. 2, Paper 18-00019, 11 pages, doi:10.1299/jamdsm.2018jamdsm0036 
[2] Vrecko, I.; Kovac, J.; Rupnik, B.; Gajsek, B. (2019). Using queuing simulation model in production process innovations, International Journal of Simulation Modelling, Vol. 18, No. 1, 47-58, doi:10.2507/IJSIMM18(1)458

[3] Habib, T.; Komoto, H. (2014). Comparative analysis of design concepts of mechatronics systems with a CAD tool for system architecting, Mechatronics, Vol. 24, No. 7, 788-804, doi:10.1016/j.mechatronics.2014.03.003

[4] Pan, Z.; Wang, X.; Teng, R.; Cao, X. (2016). Computer-aided design-while-engineering technology in top-down modelling of mechanical product, Computers in Industry, Vol. 75, 151161, doi:10.1016/j.compind.2015.05.004

[5] Sypkens Smit, M.; Bronsvoort, W. F. (2009). Integration of design and analysis models, ComputerAided Design and Applications, Vol. 6, No. 6, 795-808, doi:10.3722/cadaps.2009.795-808

[6] Unglert, J.; Hoekstra, S.; Jauregui-Becker, J.; van Houten, F. (2016). Towards decision-support for reconfigurable manufacturing systems based on computational design synthesis, Procedia CIRP, Vol. 41, 153-158, doi:10.1016/j.procir.2015.12.053

[7] Ahmed, R.; Shah, M.; Umar, M. (2016). Concepts of simulation model size and complexity, International Journal of Simulation Modelling, Vol. 15, No. 2, 213-222, doi:10.2507/ IJSIMM15(2)2.317

[8] Berselli, G.; Bilancia, P.; Luzi, L. (2020). Project-based learning of advanced CAD/CAE tools in engineering education, International Journal on Interactive Design and Manufacturing, Vol. 14, 1071-1083, doi: $10.1007 / \mathrm{s} 12008-020-00687-4$

[9] Nance, R. E.; Sargent, R. G. (2002). Perspectives on the evolution of simulation, Operations Research, Vol. 50, No. 1, 161-172, doi:10.1287/opre.50.1.161.17790

[10] Lin, C.-H.; Tung, C.-M.; Huang, C.-T. (2006). Elucidating the industrial cluster effect from a system dynamics perspective, Technovation, Vol. 26, No. 4, 473-482, doi:10.1016/ j.technovation.2004.11.008

[11] Forrester, J. W. (1993). System dynamics and the lessons of 35 years, De Greene, K. B. (Ed.), A Systems-Based Approach to Policymaking, Springer, Boston, 199-240, doi:10.1007/978-1-46153226-2 7

[12] Yang, W.-T.; Kuo, N.-W. (2003). A technique for improving readability of Forrester diagram in system dynamics perspective, Yugoslav Journal of Operations Research, Vol. 13, No. 1, 107-130, doi:10.2298/YJOR0301107Y

[13] Zupancic, B.; Sodja, A. (2013). Computer-aided physical multi-domain modelling: some experiences from education and industrial applications, Simulation Modelling Practice and Theory, Vol. 33, 45-67, doi:10.1016/j.simpat.2012.03.009

[14] Elmqvist, H. (1978). A Structured Model Language for Large Continuous Systems, PhD Thesis, Lund Institute of Technology, Lund

[15] Mahmoodi, M.; James, L. A.; Johansen, T. (2018). Automated advanced image processing for micromodel flow experiments; an application using Labview, Journal of Petroleum Science and Engineering, Vol. 167, 829-843, doi:10.1016/j.petrol.2018.02.031

[16] Pérez-Gonzalez, A.; Begovich-Mendoza, O.; Ruiz-Leon, J. (2018). Modelling of a greenhouse prototype using PSO and differential evolution algorithms based on a real-time Labview application, Applied Soft Computing, Vol. 62, 86-100, doi:10.1016/j.asoc.2017.10.023

[17] Fitzpatrick, D. E.; Ley, S. V. (2018). Engineering chemistry for the future of chemical synthesis, Tetrahedron, Vol. 74, No. 25, 3087-3100, doi:10.1016/j.tet.2017.08.050

[18] Mattsson, S. E.; Elmqvist, H.; Otter, M. (1998). Physical system modelling with Modelica, Control Engineering Practice, Vol. 6, No. 4, 501-510, doi:10.1016/S0967-0661(98)00047-1

[19] Fritzson, P. (2010). Principles of Object-Oriented Modelling and Simulation with Modelica 2.1, John Wiley \& Sons, Hoboken

[20] Li, P.; Li, Y.; Seem, J. E.; Qiao, H.; Li, X.; Winkler, J. (2014). Recent advances in dynamic modelling of HVAC equipment. Part 2: Modelica-based modelling, HVAC\&R Research, Vol. 20, No. 1, 150-161, doi:10.1080/10789669.2013.836876

[21] Nesarajah, M.; Frey, G. (2016). Multiphysics simulation in the development of thermoelectric energy harvesting systems, Journal of Electronic Materials, Vol. 45, No. 3, 1408-1411, doi:10.1007/s11664-015-4049-1 
[22] Tejado, I.; Torres, D.; Pérez, E.; Vinagre, B. M. (2016). Physical modelling based simulators to support teaching in automatic control: the rotatory pendulum, IFAC-PapersOnLine, Vol. 49, No. 6, 75-80, doi:10.1016/j.ifacol.2016.07.156

[23] Klancnik, S.; Brezocnik, M.; Balic, J. (2016). Intelligent CAD/CAM system for programming of CNC machine tools, International Journal of Simulation Modelling, Vol. 15, No. 1, 109-120, doi:10.2507/IJSIMM15(1)9.330

[24] Zheng, C.; Bricognea, M.; le Duigoua, J.; Eynarda, B. (2014). Mechatronic design process: a survey of product data model, Procedia CIRP, Vol. 21, 282-287, doi:10.1016/j.procir.2014.03.176

[25] VDI (2004). VDI 2206 - Design Methodology for Mechatronic Systems (Standard), The Association of German Engineers, Düsseldorf

[26] Chakrabarti, A.; Shea, K.; Stone, R.; Cagan, J.; Campbell, M.; Hernandez, N. V.; Wood, K. L. (2011). Computer-based design synthesis research: an overview, Journal of Computing and Information Science in Engineering, Vol. 11, No. 2, Paper 021003, 10 pages, doi:10.1115/ 1.3593409

[27] Pahl, G.; Beitz, W.; Feldhusen, J.; Grote, K. H. (2007). Engineering Design: A Systematic Approach, $3^{\text {rd }}$ edition, Springer, London 